\title{
A flow-condition-based interpolation finite element procedure for incompressible fluid flows
}

\author{
Klaus-Jürgen Bathe ${ }^{\mathrm{a}, *}$, Hou Zhang ${ }^{\mathrm{b}}$ \\ a Department of Mechanical Engineering, Massachusetts Institute of Technology, 77 Massachusetts Avenue, Room 3-356, \\ Cambridge, MA 02139, USA \\ b ADINA R\&D, Inc., 71 Elton Ave, Watertown, MA 02472, USA
}

Received 11 December 2001; accepted 18 March 2002

\begin{abstract}
A new finite element procedure for the solution of the incompressible Navier-Stokes equations is presented. In the Petrov-Galerkin formulation employed, the velocities are interpolated using the flow conditions over the elements and the pressure is interpolated to satisfy the inf-sup condition for incompressible analysis. Element control volumes are employed to satisfy local mass and momentum conservation (as in finite volume methods), which corresponds to using step functions as weight functions in the finite element method. An important achievement of the discretization scheme is that no artificial parameters are set in the scheme to reach stability for low and high Reynolds (and Péclet) number flows. The solutions of nontrivial test problems are presented to demonstrate the capability and potential of the scheme.
\end{abstract}

(C) 2002 Elsevier Science Ltd. All rights reserved.

Keywords: Finite elements; Incompressible fluid flows; High Reynolds numbers

\section{Introduction}

Finite element methods are now abundantly used in the analysis of solids and structures. The methods are employed in research and commercial computer programs for static and dynamic, linear and nonlinear analyses. Many high technology companies rely extensively on finite element analyses of their structural designs in order to reach optimum functionality and cost-effectiveness.

However, considering fluid flow analysis, the situation is quite different. While much research effort has been expended over the last three decades resulting in numerous publications on finite element methods for fluid flows, by far most fluid flow solutions in industry are still obtained using finite volume methods, see e.g.

\footnotetext{
${ }^{*}$ Corresponding author. Tel.: +1-617-253-6645; fax: +1-617253-2275.

E-mail address: kjb@mit.edu (K.J. Bathe).
}

[1,2]. Of course, researchers in finite element analysis may - and can - claim that finite volume methods are just special finite element procedures, if finite element methods are interpreted as discretization techniques in a broad sense [3].

Major reasons why industrial computer programs for fluid flow analysis are not based on the classical finite element methods, after all the research expended, are that the "traditional finite element methods" do not satisfy directly local conservation in the traditional sense and have difficulty to converge for high Reynolds number flows.

There are of course also shortcomings in the finite volume methods. Compared with finite element methods, the mathematical theory for incompressible fluid flows is less strong. However, a strong mathematical theory is needed to reach an optimal solution scheme. Optimal convergence, as the mesh is refined, is a major aim in fluid flow analysis because of the fine discretizations that frequently need be used and which lead to high cost of solutions. This shortcoming arises in part 
because interpolation functions of the field variables are not explicitly employed. The lack of these functions also means that required derivatives cannot be evaluated directly for the evaluation of the viscous terms and to set up Jacobians for the Newton-Raphson iterations. Furthermore, it is unsatisfactory that - as in the finite element method - artificial parameters to reach stability are used.

Hendriana and Bathe presented some thoughts in Ref. [4] as to what might be an "ideal" solution procedure for fluid flows. This ideal solution scheme would always give a reasonable solution to a well-posed fluid flow problem provided a reasonable mesh is used. The scheme would always converge fast in the NewtonRaphson iterations. For a coarse mesh, the solution would of course not be able to show some flow details that cannot be represented, but with refinements of the mesh, more of these details would be displayed with optimal convergence. This quality of prediction of fluid flow would hold even for flows of Reynolds numbers that would imply turbulence. Hendriana and Bathe tested various widely published finite element solution schemes and found that all of them were quite deficient when measured on this ideal solution scheme.

The objective of this paper is to present a finite element solution procedure that we developed in order to reach a scheme that is closer to this ideal solution scheme. In Ref. [4], we considered 9-node elements. We now focus on the development of a new 9-node element for two-dimensional fluid flows, but the same approach can also directly be employed for three-dimensional solutions. We regard the proposed procedure to be still a finite element discretization scheme although features of finite volume methods are employed. In the procedure, the velocities are interpolated using trial functions evaluated to incorporate the flow conditions, in the spirit of Ref. [5], which provides the "upwinding effect" in a very natural way $[6,7]$. Hence no artificial upwind parameters are used. The pressure is interpolated with the aim to satisfy the inf-sup condition for incompressible analysis, and hence here too no artificial stability constants are introduced [6,7]. For the formulation of the finite element equations, the Petrov-Galerkin method is used with step functions for the weight (test) functions over control volumes, and this results in satisfying locally the conditions of mass and momentum conservation (as in finite volume methods).

In Section 2, we first present the finite element technique, which we refer to as "a flow-condition-based interpolation", or in brief FCBI, finite element procedure. We then illustrate the performance of the procedure in the solution of some test cases with the objective to evaluate the scheme measured on the desirable characteristics of the ideal procedure mentioned above. While no detailed mathematical analysis is as yet available, based on the studies given, we can conclude already that the formulation approach is very valuable and that there is much potential in the procedure.

\section{The finite element procedure}

In this section we present the FCBI finite element procedure for the solution of the Navier-Stokes equations. We first give the mathematical model considered and then present the interpolations used. Some emphasis is given to the fact that no artificial stability constants are employed and that the conservation of mass and momentum is satisfied locally in the traditional fluid flow sense.

Throughout the paper the usual notation for Sobolev spaces is used, see e.g. [8].

\subsection{Problem formulation and discretization}

We consider a two-dimensional domain of an incompressible Navier-Stokes fluid subjected to essential and natural boundary conditions (see Fig. 1). We assume that the fluid flow problem is well-posed in the Hilbert spaces $V$ and $P$.

The differential formulation of the problem we consider is:

Find the velocity $\mathbf{v}(\mathbf{x}, t) \in V$ and pressure $p(\mathbf{x}, t) \in P$ such that

$$
\begin{aligned}
& \nabla \cdot(\rho \mathbf{v})=0 \quad(\mathbf{x}, t) \in \Omega \times[0, T] \\
& \frac{\partial \rho \mathbf{v}}{\partial t}+\nabla \cdot(\rho \mathbf{v v}-\tau)=\mathbf{0} \quad(\mathbf{x}, t) \in \Omega \times[0, T]
\end{aligned}
$$

subject to the (sufficiently smooth) initial and boundary conditions

$$
\begin{aligned}
& \mathbf{v}(\mathbf{x}, 0)={ }^{0} \mathbf{v}(\mathbf{x}), \quad p(\mathbf{x}, 0)={ }^{0} p(\mathbf{x}) \quad \mathbf{x} \in \Omega \\
& \mathbf{v}=\mathbf{v}^{s} \quad(\mathbf{x}, t) \in \bar{S}_{v} \times(0, T] \\
& \tau \cdot \mathbf{n}=\mathbf{f}^{s} \quad(\mathbf{x}, t) \in S_{f} \times(0, T]
\end{aligned}
$$

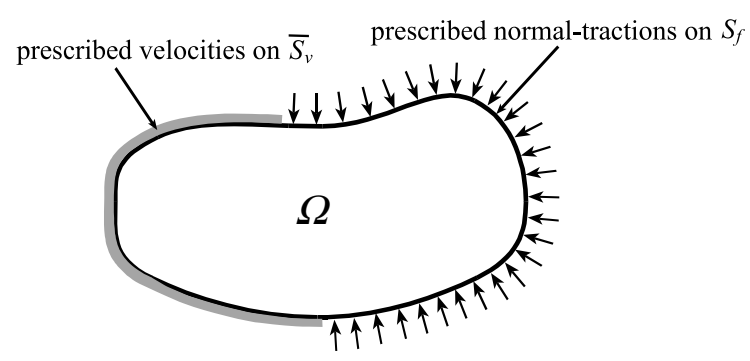

Fig. 1. Schematic of fluid flow problems considered. 
where

$\tau=\tau(\mathbf{v}, p)=-p \mathbf{I}+\mu\left[\nabla \mathbf{v}+(\nabla \mathbf{v})^{\mathrm{T}}\right]$

$\rho$ is the density, $\mu$ is the viscosity, $\Omega \in \mathbb{R}^{2}$ is a domain with the boundary $S=\bar{S}_{v} \cup S_{f}\left(S_{v} \cap S_{f}=\emptyset\right), T$ is the time span considered, $\mathbf{v}^{s}$ are the prescribed velocities on the boundary $\bar{S}_{v}, \mathbf{f}^{s}$ are the prescribed tractions on the boundary $S_{f}$ and $\mathbf{n}$ is the unit normal to the boundary.

We note that we set out to solve the "conservative form" of the Navier-Stokes equations [7]. The reason is that we want to satisfy the local conservation of mass and momentum in the classical sense.

Our objective is to develop a solution procedure for the Navier-Stokes equations that is close to the ideal scheme mentioned in Section 1, see also Ref. [4]. As is well known, the Navier-Stokes equations are self-consistent up to very high Reynolds numbers and weak solutions exist, provided of course transient analysis conditions are considered and the boundary and initial conditions are sufficiently smooth $[9,10]$. Hence, it is reasonable to require that a numerical solution scheme should be able to solve flow conditions at very high Reynolds numbers.

For the finite element solution, we use a PetrovGalerkin variational formulation with subspaces $V_{h}, U_{h}$ and $W_{h}$ of $V$, and $P_{h}$ and $Q_{h}$ of $P$ of the problem in Eqs. (1)-(6). The formulation used is:

Find $\mathbf{v} \in V_{h}, \mathbf{u} \in U_{h}, p \in P_{h}$ such that for all $w \in W_{h}$ and $q \in Q_{h}$

$$
\begin{aligned}
& \int_{\Omega} w\left[\frac{\partial \rho \mathbf{u}}{\partial t}+\nabla \cdot(\rho \mathbf{u v}-\tau(\mathbf{u}, p))\right] \mathrm{d} \Omega=\mathbf{0} \\
& \int_{\Omega} q \nabla \cdot(\rho \mathbf{u}) \mathrm{d} \Omega=0
\end{aligned}
$$

To define the spaces used in the formulation, consider Fig. 2, where we show a mesh of elements in their natural coordinate systems. To obtain the matrices correspond- ing to a general two-dimensional geometry, the usual isoparametric transformations are used [7]. The figure shows a patch of typical 9-node elements, see Fig. 2(a), and a "sub-element", see Fig. 2(b). This sub-element is defined by four nodes of the 9-node element and is used for the interpolation of velocities. Each 9-node element is thought of to consist of four 4-node sub-elements.

For the definition of the space $U_{h}$, we refer to the subelement. The trial functions in $U_{h}$ are defined as

$\left[\begin{array}{ll}h_{1}^{u} & h_{4}^{u} \\ h_{2}^{u} & h_{3}^{u}\end{array}\right]=\mathbf{h}(\xi) \mathbf{h}^{\mathrm{T}}(\eta)$

where $\mathbf{h}^{\mathrm{T}}(y)=[1-y, y] \quad(y=\xi, \eta \quad$ with $\quad 0 \leqslant \xi, \eta \leqslant 1)$. Similarly, an element in the space $P_{h}$ is given by (refer to Fig. 2(a))

$\left[\begin{array}{ll}h_{1}^{p} & h_{4}^{p} \\ h_{2}^{p} & h_{3}^{p}\end{array}\right]=\mathbf{h}(r) \mathbf{h}^{\mathrm{T}}(s)$

with $0 \leqslant r, s \leqslant 1$.

The trial functions in $V_{h}$ are defined using the flow conditions along each side of the sub-element. The functions are, for the flux through $a b$,

$\left[\begin{array}{ll}h_{1}^{v} & h_{4}^{v} \\ h_{2}^{v} & h_{3}^{v}\end{array}\right]=\left[\mathbf{h}\left(x^{1}\right), \mathbf{h}\left(x^{2}\right)\right] \mathbf{h}(\eta) \mathbf{h}^{\mathrm{T}}(\eta)$

with

$x^{k}=\frac{\mathrm{e}^{\mathrm{e}^{k} \xi}-1}{\mathrm{e}^{q^{k}}-1}, \quad q^{k}=\frac{\rho \overline{\mathbf{u}}^{k} \cdot \Delta \mathbf{x}^{k}}{\mu}$

where $\overline{\mathbf{u}}^{k} \in U_{h}$ and is the velocity at the center of the sides considered $(\xi=1 / 2$ and $\eta=0,1$ for $k=1,2$ respectively). Analogously, the functions are constructed for the flux through $b c$, and so on.

Note that these functions satisfy the requirement $\sum h_{i}=1$, although different flow conditions may be present at the element edges [7].

The elements in the space $Q_{h}$ are step functions. Referring to Fig. 2(a), we have, at node 2, for example,

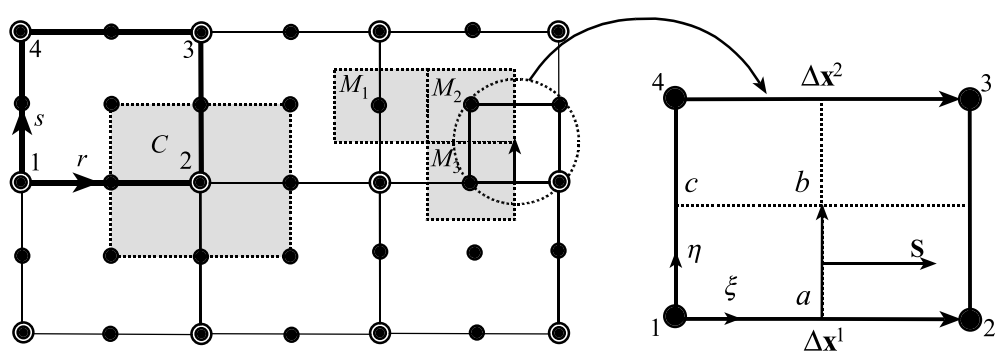

(a) 9-node elements

(b) sub-element

Fig. 2. 9-Node elements and a sub-element in isoparametric coordinates. Domains over which the (constant) weight functions are defined. 
$h_{2}^{q}= \begin{cases}1 & (r, s) \in\left[\frac{1}{2}, 1\right] \times\left[0, \frac{1}{2}\right] \\ 0 & \text { else }\end{cases}$

Similarly, the weight functions in the space $W_{h}$ are also step functions. Considering the sub-element we have at node 1 , for example,

$h_{1}^{w}= \begin{cases}1 & (\xi, \eta) \in\left[0, \frac{1}{2}\right] \times\left[0, \frac{1}{2}\right] \\ 0 & \text { else }\end{cases}$

Remark 1. The fact that two spaces for the trial functions are used needs a comment. We could use only the space $V_{h}$ (and hence substitute $\mathbf{u}$ by $\mathbf{v}$ in Eq. (7)), but then clearly the discrete finite element equations would become even more nonlinear, because the trial functions in $V_{h}$ contain the exponential expressions. Therefore, we use both spaces $U_{h}$ and $V_{h}$, but of course the functions in these spaces are "attached" to the same nodal velocity variables. For example, the velocities $\mathbf{v}$ and $\mathbf{u}$ in Fig. 2 are attached to the trial functions in $U_{h}$ and $V_{h}$ by

$\mathbf{v}=h_{i}^{v} \mathbf{v}_{i}$

$\mathbf{u}=h_{i}^{u} \mathbf{v}_{i}$

where $\mathbf{v}_{i}$ are the nodal velocity variables.

The rational for proceeding in this way lies in that it is $\mathbf{v}$ in Eq. (7) which introduces the instability in the numerical solution and which therefore needs to be interpolated exponentially. Another reason is that we want the scheme to be applicable to any transport equation, for example, the advection-diffusion equation where, in the convective term, the temperature would be interpolated in $V_{h}$ and the velocity in $U_{h}$.

Remark 2. We should note that the evaluation of Eqs. (7) and (8) with the given functions reduces to an evaluation of the integrals around the control volumes shown in Fig. 2(a). Considering Eq. (7), we have one control volume for each finite element node in the element assemblage (see control volumes $M_{1}, M_{2}$ and $M_{3}$ ), and considering Eq. (8) we have one control volume for each pressure node in the element assemblage (see control volume $C$ ). The use of these control volumes enforces the local conservation of momentum and mass, respectively.

Remark 3. The finite element procedure given here is related to finite volume methods [1,11], discontinuous finite element methods $[12,13]$ and the use of bubbles [14]. Our objective in constructing the FCBI procedure is to synthesize ideas in order to obtain a solution procedure closer to the ideal scheme described in Ref. [4], and a scheme that mathematically can be analyzed such that further ideas of improvements will come forth.

\subsection{On the conservation of mass and momentum}

In this section we endeavor to discuss the important properties of conservation of mass and momentum and in which way these conservation conditions are satisfied. We consider first the traditional finite element methods and then our procedure. We refer to "flux conservation" because the essence of the conditions is to conserve the "flux" as imposed through the divergence operator in Eqs. (1) and (2) (the mass flux in the first equation and the momentum flux in the second equation).

The finite element methods for fluid flows were developed because a great success of finite element procedures was seen in structural analysis. For the analysis of structures, Lagrangian formulations and the principle of virtual work are used to obtain the well-known finite element equations

$\mathbf{F}=\mathbf{R}$

where $\mathbf{R}$ is a vector of all externally applied nodal forces and $\mathbf{F}$ is a vector equivalent (in the virtual work sense) to the element internal stresses. The details of derivation of these equations are widely available, but we should note that the nodal force vector $\mathbf{R}$ contains the contributions of all externally applied forces, including the contributions from surface tractions, body forces, concentrated loads, initial stresses, and in transient analysis inertia forces. Of course, Eq. (17) is applicable in linear and nonlinear analyses. The vector $\mathbf{R}$ is assembled by summing over all element contributions, and similarly, the vector $\mathbf{F}$ is obtained as

$\mathbf{F}=\sum_{m} \mathbf{F}^{(m)}$

where $\mathbf{F}^{(m)}$ lists the nodal forces equivalent to the element stresses of element $m$. Using the notation of Ref. [7], we have

$\mathbf{F}^{(m)}=\int_{V^{(m)}} \mathbf{B}^{(m) \mathrm{T}} \boldsymbol{\tau}^{(m)} \mathrm{d} V^{(m)}$

where $\mathbf{B}^{(m)}$ is the strain displacement matrix of element $m, \tau^{(m)}$ is the stress in element $m$, and we are integrating over the element volume. While the above relations are written for the commonly used displacement-based finite element methods, in order to focus on the essence of the discussion, the same equations are also fundamental when considering mixed methods [7].

Considering Eqs. (17)-(19), we can directly infer (prove) that in finite element analysis the following two fundamental Properties I and II are satisfied:

Property I (Nodal point equilibrium). At each node in the element assemblage, the sum of the element forces $\mathbf{F}^{(m)}$ is equal to the externally applied forces listed in $\mathbf{R}$. This property of course directly follows from Eq. (17), but we 
must recall that at those nodes where displacements are prescribed, the reactions are calculated from the summation of the element internal forces.

Property II (Element equilibrium). Each element $m$ is in equilibrium under its forces $\mathbf{F}^{(m)}$. This property follows directly from Eq. (19) and holds for any properly formulated finite element. The proof is simple and based on subjecting the element $m$ to rigid body translations and rotations.

These properties are illustrated in Fig. 3 and hold of course for any coarse or fine mesh. For details on these properties and a demonstrative example, see Ref. [7, pp. 177-182].

The above equilibrium properties express fundamental requirements that structural engineers are used to work with. If only nodal point concentrated loads are applied to a truss or beam model, the exact solutions to the mathematical models are directly obtained (if applied distributed loads are present, special techniques can be used that then also lead to exact solutions). However, in the general finite element analysis of solids, plates or shells, of course, only approximate solutions are obtained. Specifically, consider the widely used displacement-based finite element discretization. Although the conditions of compatibility and stress-strain relationships are fulfilled, and the equilibrium Properties I and II are satisfied, differential equilibrium (within the elements and on the boundary) is in general not satisfied. This results into stress jumps between elements and the fact that the externally applied body forces and surface tractions are not balanced by the finite element internal

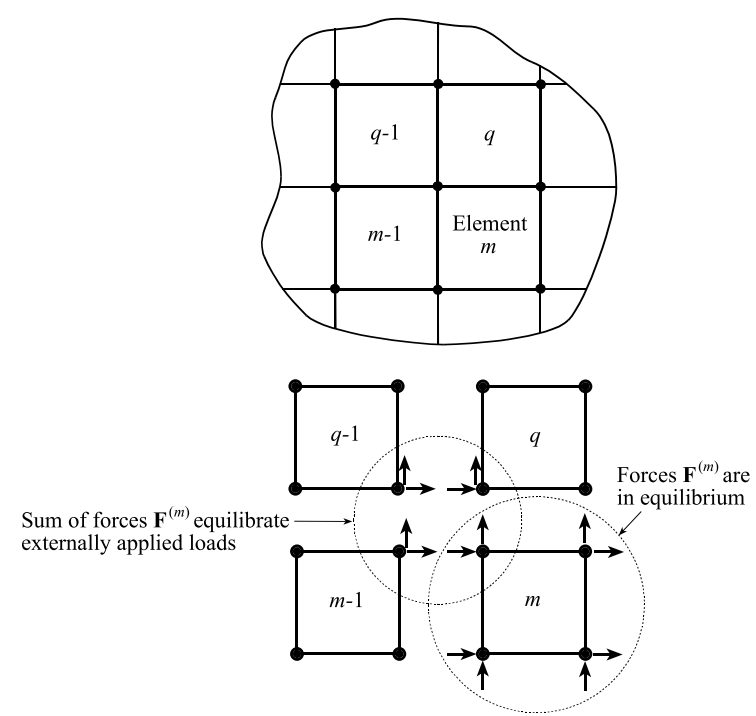

Fig. 3. Nodal point and element equilibrium in a finite element analysis (taken from Ref. [7]). stresses. Of course, as is well-known, these errors diminish sufficiently as the mesh is refined [7].

Consider next the generic fluid flow problem, schematically shown in Fig. 1, and governed by the NavierStokes equations (1)-(6). If the standard finite element discretization is carried out, algebraic equations of the forms of Eqs. (17)-(19) are also obtained but of course for the nodal fluxes. Properties I and II are also applicable - but naturally for the nodal fluxes [7]. Hence, "nodal flux equilibrium" as schematically shown in Fig. 3 on the assemblage nodal level and element level is satisfied.

The conditions fulfilled in traditional finite element fluid flow analyses are therefore very similar to those in structural analysis. However, whereas in structural analysis, the conditions of "force equilibrium" expressed in Properties I and II are sufficient to satisfy structural engineers, the "flux equilibrium" alone, expressed also in Properties I and II, is generally not sufficient to satisfy an analyst of fluid flow problems. Here recall that using the Lagrangian formulation for structural analysis, mass conservation is automatically satisfied. However, in Eulerian formulations of fluid flows, flux conservation for any sub-domain (of finite elements or finite volumes) in the traditional sense (integrating around the subdomain boundary) needs to be fulfilled, and this property is of course what finite volume methods are based on and hence always directly satisfy. Of course, standard finite element schemes can be extended with special techniques such as post-processing methods in order to more closely satisfy flux equilibrium (in the control volume sense) but such methods add to the complexity and cost of solution. Also, finite element methods with discontinuous weight functions ( $w$ in Eq. (7)) can be used and this is what we have selected to pursue in our developments.

Considering the FCBI finite element procedure presented above, since the weight functions are chosen as the given step functions, flux conservation is satisfied in the control volume sense over the sub-domains shown in Fig. 2(a). Hence the Property I can be restated as follows:

Property I for FCBI scheme (Control volume "flux equilibrium"- that is, mass and momentum conservation). The FCBI finite element solution procedure satisfies local mass and momentum conservation for the control volumes used to construct the algebraic finite element equations.

This property is an important characteristic of the solution scheme because of the theoretical and practical need to satisfy locally conservation of mass and momentum. The property is probably also, in part, the reason why reasonable solutions of fluid flow problems with very high Reynolds numbers could be obtained with relatively coarse meshes (see Section 3). 


\section{Example solutions}

The objective in this section is to give the results obtained in the solution of some fluid flow problems when using the FCBI procedure. Of course, for the simple one-dimensional advection-diffusion equation, frequently solved when evaluating a procedure [7], the exact solution is obtained for any mesh used (see e.g. Fig. 4). We next consider additional problems and focus on the aim that we would like to obtain reasonable solutions for well-posed problems even when a flow of high Reynolds number is considered and only a coarse mesh is used.

\subsection{Solution of driven cavity flow}

The cavity flow problem shown in Fig. 5 has been used extensively to test computational fluid dynamics schemes. We used two meshes to solve this problem for various Reynolds numbers: an 8 by 8 mesh, the "coarse mesh", and a 24 by 24 mesh, called the "fine mesh", (although this is really not yet a fine mesh for the problem).

Fig. 6 gives the velocity solution results for Reynolds numbers 1, 100, 10,000 and 1,000,000 using the coarse and fine meshes. We see that reasonable results have been obtained with all meshes, with the fine mesh showing more details in the flows. Of course, as the Reynolds number increases, the predicted flow solution hardly changes from a certain high Reynolds number onwards (which depends on the mesh used).

For further evaluation we also solved the case of $\mathrm{Re}=1000$ using the coarse, fine and in addition a very fine mesh (of 100 by 100 elements). The results are

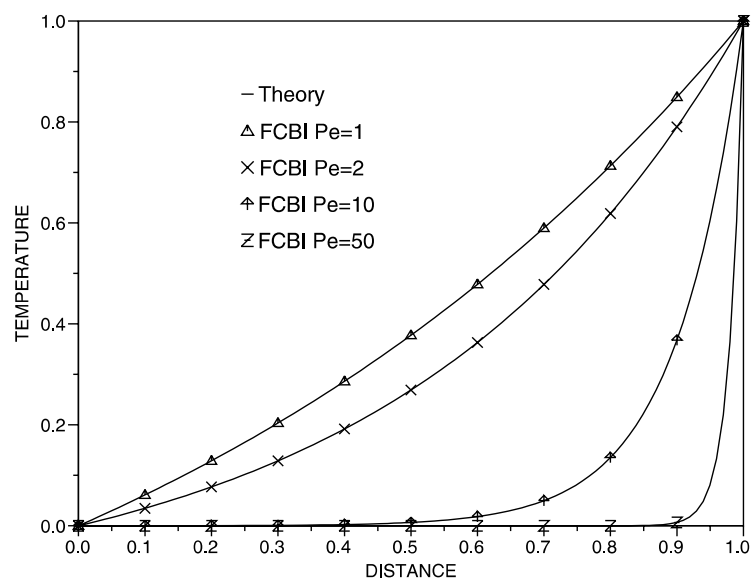

Fig. 4. Numerical solutions of one-dimensional advection-diffusion equation compared with exact solutions at various Péclet numbers. Five elements are used. (For problem definition, see [7, p. 683].)
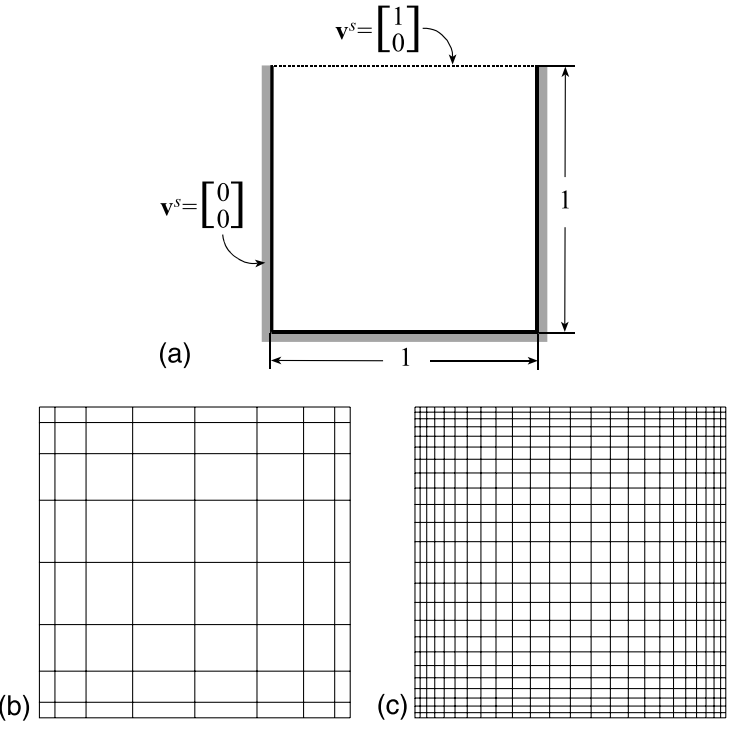

Fig. 5. Driven cavity flow problem and meshes used: (a) problem definition; (b) coarse mesh and (c) fine mesh.

compared with the solution of Ghia et al. [15] in Fig. 7, which is considered to be an accurate solution. We see that the results obtained with the coarse and fine meshes are reasonable but of course not accurate, and that the results given by the very fine mesh are accurate. This convergence is of course also a required quality of a solution scheme.

\subsection{Solution of $S$-channel flow}

We consider next the solution of the S-channel flow shown in Fig. 8. This problem was already used to test solution schemes in Ref. [4], and is a difficult problem to solve when higher Reynolds number flows are considered. The difficulties arise because the corners in the geometry force changes in flow directions and a circulation exists for higher Reynolds numbers in the horizontal exit section. In this case we use the "coarse mesh" and the "fine mesh" shown in Fig. 8.

Figs. 9 and 10 show the velocity and pressure solutions obtained for Reynolds numbers 1, 100, 10,000 and $1,000,000$ using the coarse and fine meshes. As in the cavity flow problem, we see that reasonable results have been obtained in all cases, and of course using the fine mesh more details in the flows are calculated. In particular, the flow circulation near the outlet is predicted using the fine mesh. Also, from a certain Reynolds number onwards (which depends on the mesh) the predicted flow solution hardly changes.

Fig. 11 compares the pressure solutions for the case of $\mathrm{Re}=100$ obtained using the FCBI scheme (with the coarse and fine meshes) and the standard Galerkin 9/4-c 


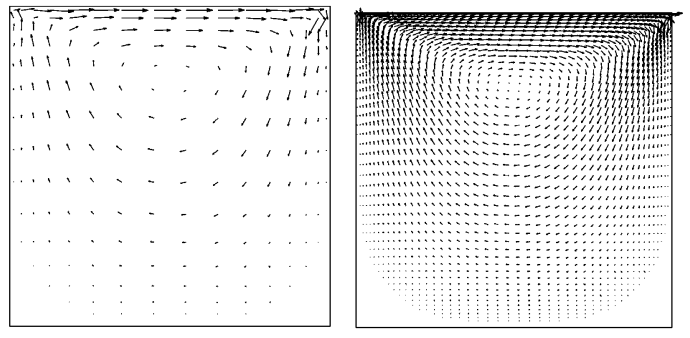

(a) $\operatorname{Re}=1$

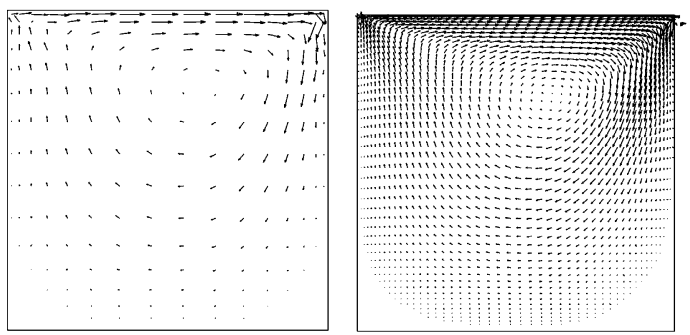

(b) $\operatorname{Re}=10^{2}$

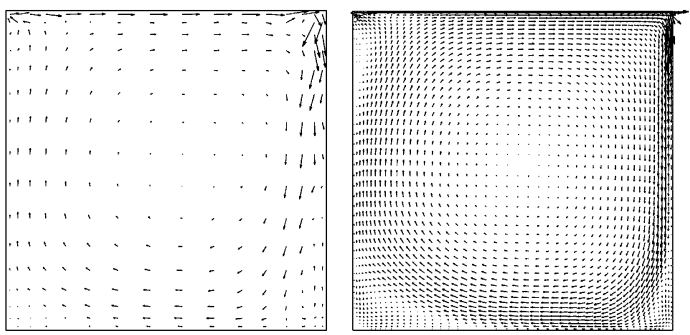

(c) $\operatorname{Re}=10^{4}$

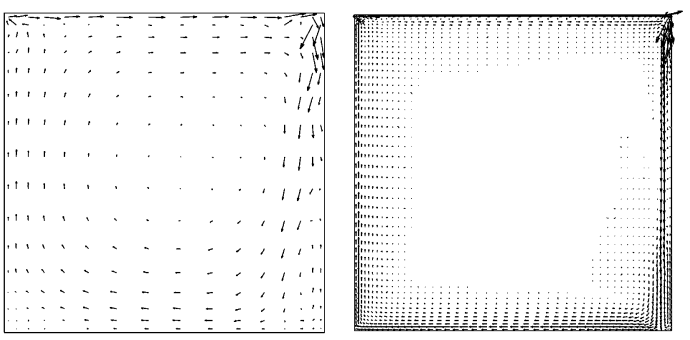

(d) $\operatorname{Re}=10^{6}$

Fig. 6. Velocity solutions at Reynolds numbers 1 (a), $10^{2}$ (b), $10^{4}$ (c) and $10^{6}$ (d) in driven cavity flow problem.

element (with only the fine mesh and no upwinding) [7]. We notice that the solutions using the FCBI scheme compare well with the solution obtained using the 9/4-c element.

\subsection{Fluid flow interacting with cantilever}

An important objective in the development of the new finite element procedure is to be able to solve problems of fluid flows fully interacting with flexible structures. In such solutions, the structure may undergo

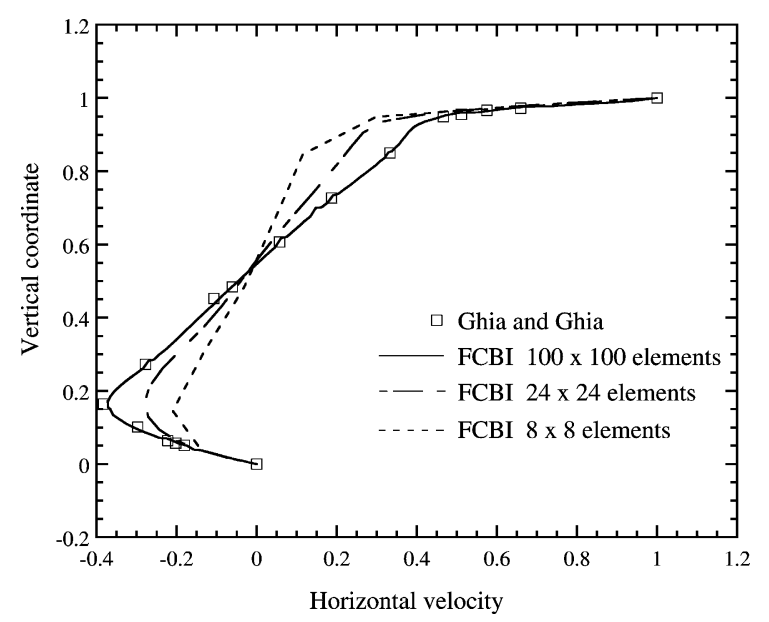

Fig. 7. Horizontal velocity along the vertical centerline of the cavity $(\mathrm{Re}=1000)$.

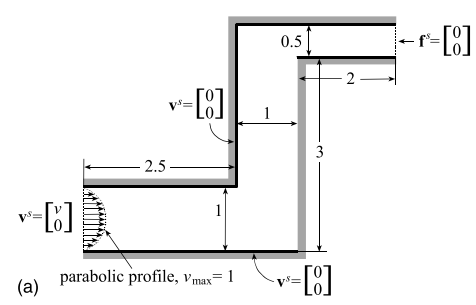

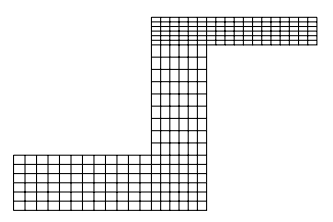

(b)

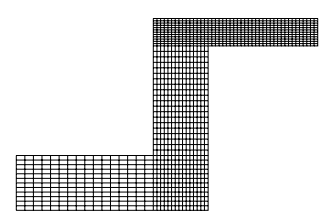

(c)
Fig. 8. S-channel flow problem and meshes used: (a) problem definition; (b) coarse mesh and (c) fine mesh.

very large displacements. The structure and fully coupled fluid flow then correspond to a highly nonlinear system, and it is important that a stable and accurate solution procedure for the fluid flow be used [16].

Fig. 12 shows a rather simple problem, taken from the ADINA verification manual, which we use to illustrate the FCBI solution capability. The problem was solved using coarse and fine meshes for the fluid, whereas just a coarse mesh was employed for the cantilever. The arbitrary Lagrangian-Eulerian (ALE) formulation available in ADINA was used [17]. Figs. 13 and 14 give the calculated velocity and pressure distributions, together with the deformations, drawn to scale, of the cantilever. We note that the solution results using the coarse mesh are of course different from the results obtained using the fine mesh, but the difference is reasonable. 

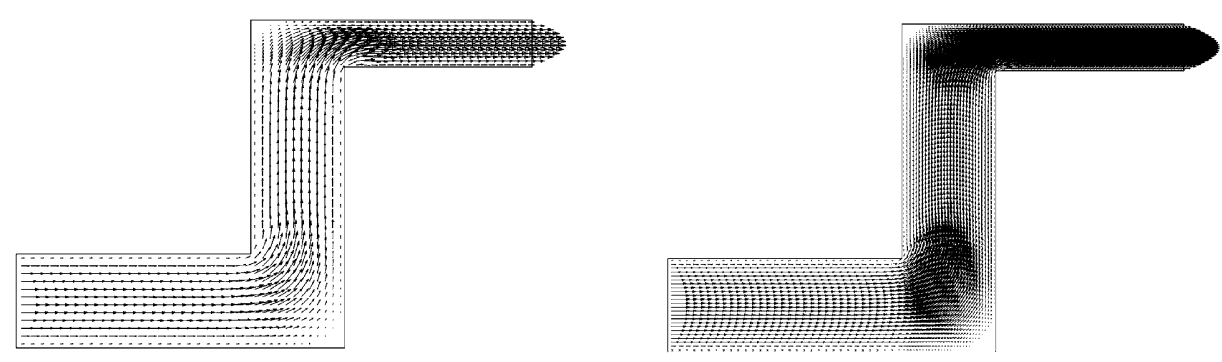

(a) $\operatorname{Re}=1$
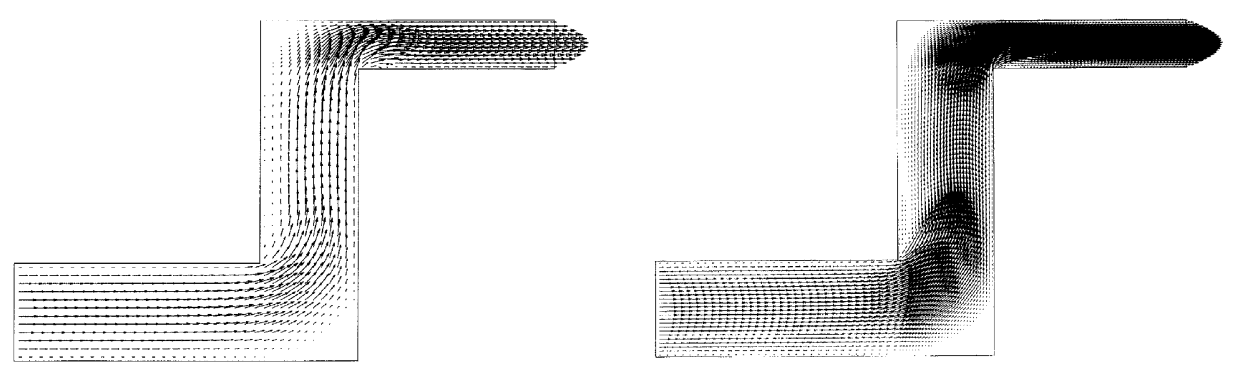

(b) $\operatorname{Re}=10^{2}$
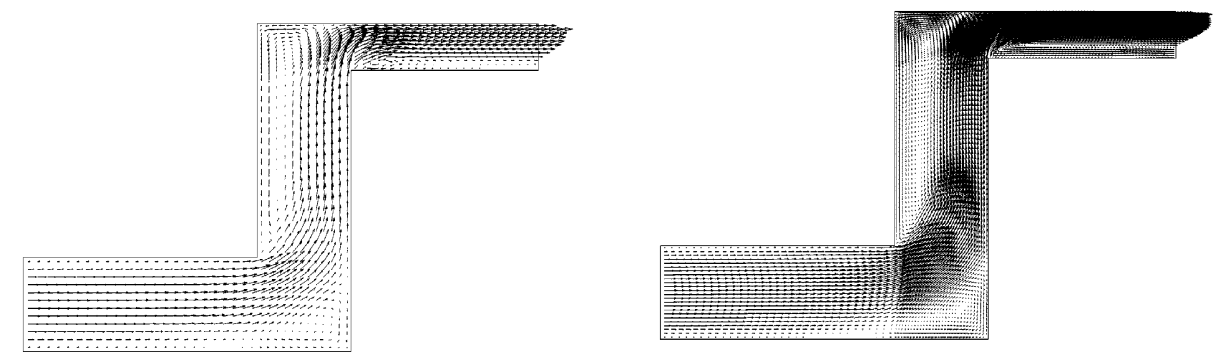

(c) $\operatorname{Re}=10^{4}$
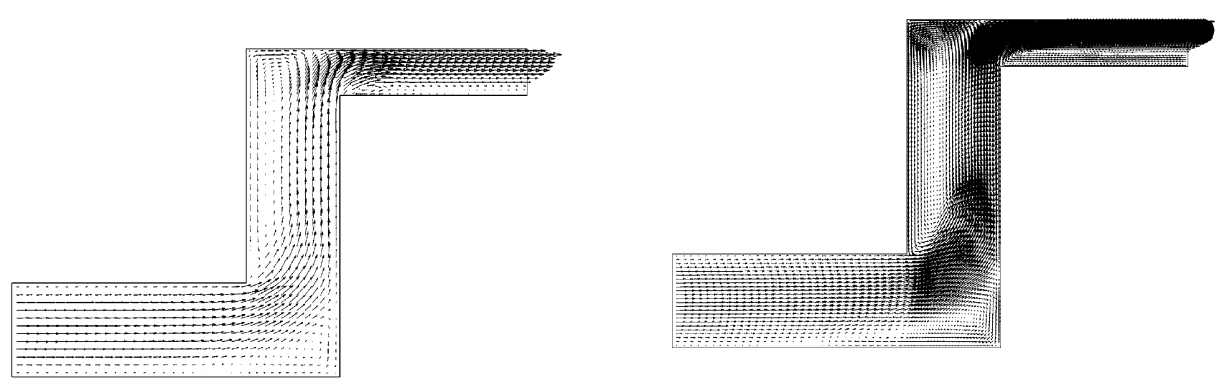

(d) $\operatorname{Re}=10^{6}$

Fig. 9. Velocity solutions at Reynolds numbers 1 (a), $10^{2}$ (b), $10^{4}$ (c) and $10^{6}$ (d) in S-channel flow problem.

\section{Concluding remarks}

The objective of this paper was to present a finite element solution scheme for incompressible fluid flows, which we have developed in our aim to obtain a solution procedure closer to the ideal scheme discussed in Ref. [4]. The solution procedure is based on a Petrov-Galerkin formulation for a 9-node element, in which an FCBI 

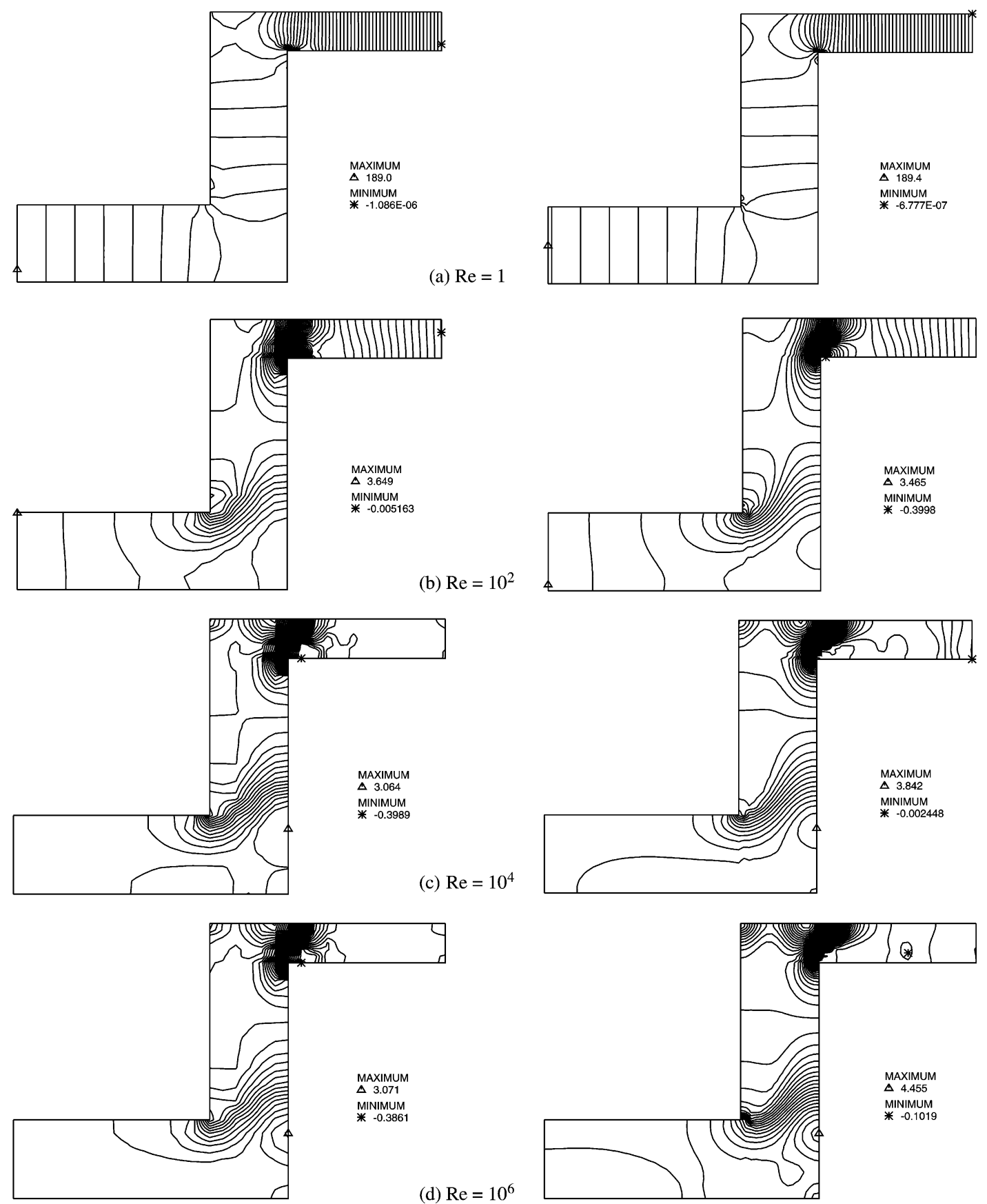

Fig. 10. Pressure solutions at Reynolds numbers 1 (a), $10^{2}$ (b), $10^{4}$ (c) and $10^{6}$ (d) in S-channel flow problem.

procedure of the velocities is used to provide the effect of upwinding. Also, step weight functions, in essence, result in using control volumes for mass and momentum conservation. The pressure is bi-linearly interpolated as for the 9/4-c (Q2-Q1) element [7]. No artificial stabilization parameters are used in the formulation.
The element has been employed to solve some low and high Reynolds number test problems. Coarse and (reasonably) fine meshes were used in the solutions. With these meshes, accurate solutions have been obtained for flows of moderate Reynolds numbers. The important quality of the solution scheme displayed in 

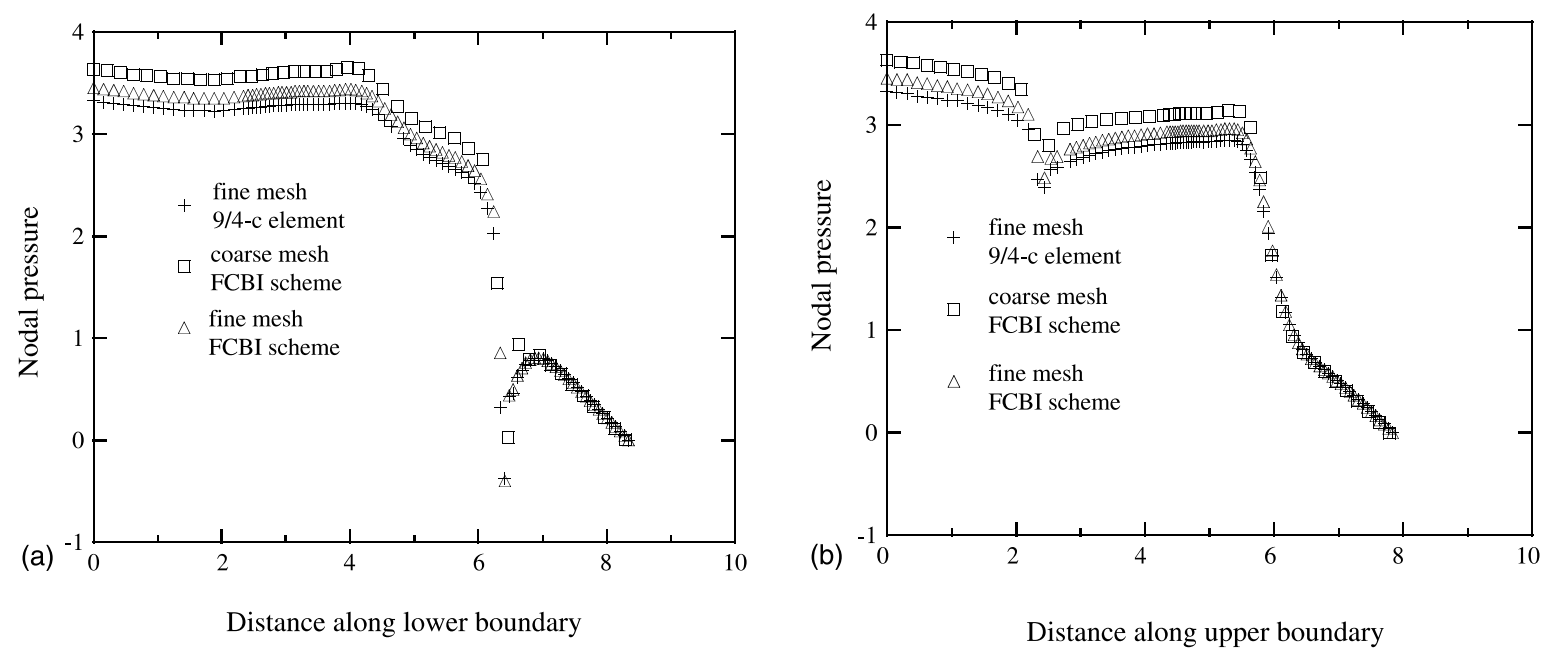

Fig. 11. Comparison of predicted pressures in solutions of S-channel flow problem $(\mathrm{Re}=100)$.

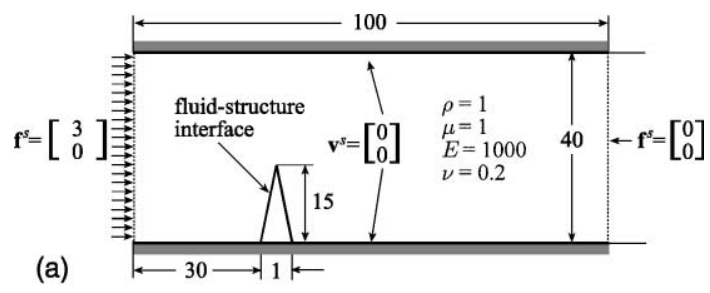

(b)

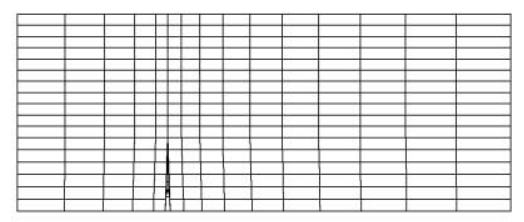

(c)

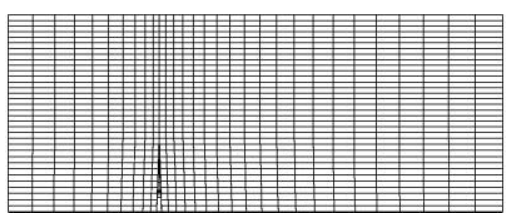

Fig. 12. Fluid flow interacting with cantilever and meshes used: (a) problem definition; (b) coarse fluid and solid mesh and (c) fine fluid mesh and the same coarse solid mesh.

the results, however, is that reasonable flow solutions could be obtained up to the Reynolds number of one million. Of course, no details of the actual and very complex flows can be resolved with the meshes used when the Reynolds number is very high.

The solution approach using the FCBI scheme shows much promise, but significant further developments are necessary. The fact that a reasonable flow solution is obtained is encouraging but of course an accurate so-

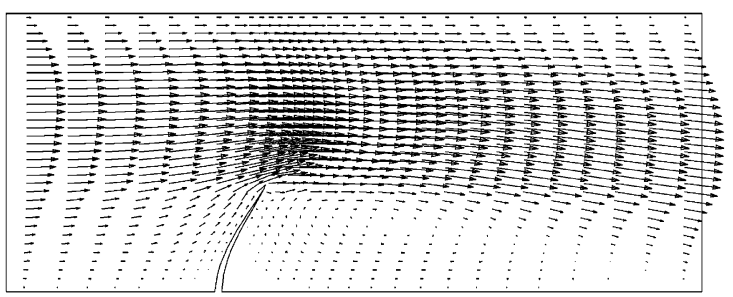

(a)

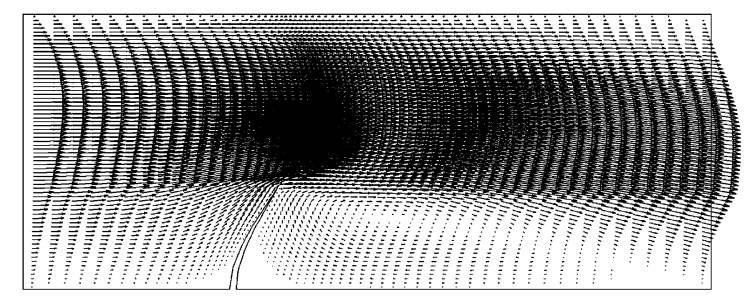

(b)

Fig. 13. Velocity solutions using coarse mesh (a) and fine mesh (b) in the problem of fluid flow interacting with cantilever.

lution is needed; an ideal solution procedure would be stable and optimal in terms of an appropriate error measure.

The element presented in this paper is not a highorder accurate element due to the interpolations used. Achieving a higher order of convergence, while keeping the desirable property of the ideal scheme to be able to solve high Reynolds number flows, is a challenge for further development of the procedure.

Clearly, deeper analyses and more numerical testing of the scheme will guide us in improving the method, but it appears that considerable potential exists in arriving at an effective solution procedure that is close to the ideal 

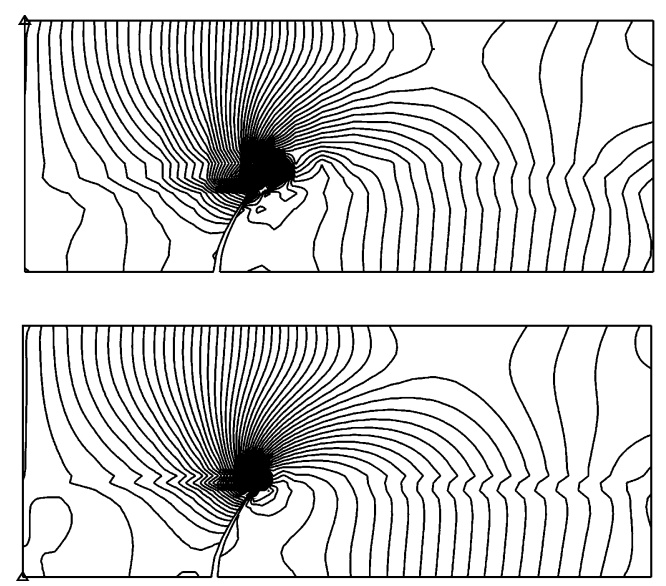

(a)

Fig. 14. Pressure solutions using coarse mesh (a) and fine mesh (b) in the problem of fluid flow interacting with cantilever.

solution scheme of Ref. [4]. Also, the approach used should be very valuable for developing effective solution schemes for compressible flows.

\section{References}

[1] Bathe KJ (Ed), Computational Fluid and Solid Mechanics, Proceedings of the First M.I.T. Conference on Computational Fluid and Solid Mechanics, Elsevier Science, 2001.

[2] Lockwood FC, Abbas T, Kandamby NH, Sakthitharan V. CFD experience on industrial combustors. Prog Comput Fluid Dyn 2001;1(1-3):1-13.

[3] Idelsohn SR, Oñate E. Finite volumes and finite elements-two good friends. Int $\mathbf{J}$ Numer Meth Engng 1994;37(19):3323-41.

[4] Hendriana D, Bathe KJ. On upwind methods for parabolic finite elements in incompressible flows. Int J Numer Meth Engng 2000;47:317-40.
[5] Bathe KJ, Pontaza J. A flow-condition-based interpolation mixed finite element procedure for higher Reynolds number fluid flows. Math Models Meth Appl Sci 2002;12(4): $1-15$.

[6] Bathe KJ. The inf-sup condition and its evaluation for mixed finite element methods. Comput Struct 2001;79:24352, 971.

[7] Bathe KJ. Finite element procedures. Prentice-Hall; 1996.

[8] Lions JL, Magenes E. Nonhomogeneous boundary value problems and applications, vol. 1. Springer-Verlag; 1972.

[9] Doering CR, Gibbon JD. Applied analysis of the NavierStokes equations. Cambridge University Press; 1995.

[10] Rauh A. Remarks on unsolved basic problems of the Navier-Stokes equations. In: Proceedings of the Conference Let's Face Chaos through Nonlinear Dynamics, Maribor, Slovenia. June 24-July 5, 1996.

[11] Minkowycz WJ, Sparrow EM, Schneider GE, Pletcher RH. Handbook of numerical heat transfer. John Wiley; 1988.

[12] Arnold DN, Brezzi F, Cockburn B, Marini LD. Unified analysis of discontinuous Galerkin methods for elliptic problems. SIAM J Num Anal, in press.

[13] Cockburn B, Karniadakis GE, Shu CW, editors. Discontinuous Galerkin methods - theory, computation and applications. Lecture Notes in Computational Science and Engineering, Vol. 11, Springer-Verlag, 2000.

[14] Brezzi F, Marini LD. Subgrid phenomena and numerical schemes. In: Proceedings of the International Symposium on Mathematical Modeling and Numerical Simulation in Continuum Mechanics, Yamaguchi. September 29-October 3, 2000 .

[15] Ghia U, Ghia KN, Shin CT. High-Re solutions for incompressible flow using the Navier-Stokes equations and a multigrid method. J Comp Phys 1982;48:387411.

[16] Rugonyi S, Bathe KJ. On finite element analysis of fluid flows fully coupled with structural interactions. Comput Model Engng Sci 2001;2:195-212.

[17] Bathe KJ, Zhang H, Ji S. Finite element analysis of fluid flows fully coupled with structural interactions. Comput Struct 1999;72(1-3):1-16. 